\title{
Antibacterial activity of amino- and amido- terminated poly (amidoamine)-G6 dendrimer on isolated bacteria from clinical specimens and standard strains
}

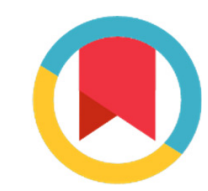

\begin{abstract}
Ayoob Rastegar ${ }^{1,2}$, Shahram Nazari*2, Ahmad Allahabadi ${ }^{1}$, Farahnaz Falanji ${ }^{3}$, Fakhreddin Akbari Dourbash ${ }^{4}$, Zahra Rezai $^{1}$, Soudabeh Alizadeh Matboo ${ }^{5}$, Reza Hekmat-Shoar ${ }^{6}$, Seyed Mohsen Mohseni ${ }^{7}$, Gharib Majidi ${ }^{8}$
\end{abstract}

Received: 8 July 2016

Published: 24 Sep 2017

\section{Abstract}

Background: Nanoscale poly (amidoamine) dendrimers have been investigated for their biological demands, but their antibacterial activity has not been widely discovered. Thus, the sixth generation of poly (amidoamine) dendrimer (PAMAM-G6) was synthesized and its antibacterial activities were evaluated on Gram-negative bacteria; P. aeruginosa, E. coli, A. baumannii, S. typhimurium, S. dysenteriae, K. pneumoniae, P. mirabilis, and Gram-positive bacteria, and S.aureus and B. subtilis, which were isolated from different clinical specimens and standard strains of these bacteria.

Methods: In this study, 980 specimens including urine (47\%), blood (27\%), sputum (13\%), wounds $(8 \%)$, and burns $(5 \%)$ were collected from clinical specimens of 16 hospitals and clinics in city of Sabzevar, Iran. Then, the target bacteria were isolated and identified using standard methods. Minimum inhibitory concentration and minimum bactericidal concentrations against Gram-positive and Gram-negative bacteria were determined according to guidelines described by clinical and laboratory standards institute (CLSI). Standard discs were prepared using $0.025,0.25,2.5$, and $25 \mu \mathrm{g} / \mathrm{mL}$ concentrations of PAMAM-G6 on Mueller-Hinton agar plates to determinate the zone of inhibition. The cytotoxicity of PAMAM-G6 dendrimer was evaluated in HCT116 cells by MTT assay.

Results: The most important isolated bacteria were E. coli (23.65\%), S. aureus (24.7\%), P. aeruginosa (10.49\%), B. subtilis (7.7\%), S. typhimurium (8.87\%), A. baumannii (7.02\%), K. pneumoniae (7.1\%), P. mirabilis (6.46\%), and S. dysenteriae (3.6\%). Moreover, it was found that poly (amidoamine)-G6 exhibited more antibacterial efficacy on standard strains than isolated bacteria from clinical samples $(\mathrm{p}<0.05)$. The cytotoxicity of PAMAM-G6 to the cells showed that cytotoxicity depended on the concentration level and exposure time.

Conclusion: The PAMAM-G6 dendrimer showed a positive impact on the removal of dominant bacterial isolated from clinical specimens and standard strains.

Keywords: Antimicrobial resistance, Cytotoxicity, Poly (amidoamine)-G6, Health care-associated infections, Nosocomial pathogens, Novel antibacterial, Synthesis

Copyright@ Iran University of Medical Sciences

Cite this article as: Rastegar A, Nazari Sh, Allahabadi A, Falanji F, Akbari Dourbash F, Rezai Z, Alizadeh Matboo S, Hekmat-Shoar R, Mohseni SM, Majidi Gh. Antibacterial activity of amino- and amido- terminated poly (amidoamine)-G6 dendrimer on isolated bacteria from clinical specimens and standard strains. Med J Islam Repub Iran. 2017 (24 Sep);31:64. https://doi.org/10.14196/mjiri.31.64

\section{Introduction}

Health care-associated infections (HCAI) are a common cause of morbidity and mortality and are considered to be one of the most adverse events in health care. Also, these

Corresponding author: Dr Shahram Nazari, shahramnazari73@yahoo.com

1. Department of Environmental Health Engineering, School of Public Health, Sabzevar University of Medical Sciences, Sabzevar, Iran.

2. Student Research committee, Faculty of Public Health Branch, Iran University of Medical Sciences, Tehran, Iran.

3. Cellular and Molecular Research Center, Sabzevar University of Medical Sciences, Sabzevar, Iran.

4. Department of Materials science and Engineering, Tarbiat Modares University, Tehran, Iran.

5. School of Public Health, Ardabil University of Medical Sciences, Ardabil, Iran.

6. Department of Occupational Health Engineering, School of Public Health, Sabzevar University of Medical Sciences, Sabzevar, Iran.

7. Department of Environmental Health Engineering, School of Public Health, Shahid Beheshti University of Medical Sciences, Tehran, Iran.

${ }^{8}$ Research Center for Environmental Pollutants, Qom University of Medical Sciences, Qom, Iran. infections prolong hospitalization, require further extensive diagnostics and treatment, and are associated with additional costs (1). The failure of treatment for these in-

$\uparrow$ What is "already known" in this topic:

Health care associated infection (HCAI) presents a major problem for patient safety and could lead to prolonged hospitalization, long-term disability, high costs for patients and their families, and excess deaths.

\section{$\rightarrow$ What this article adds:}

Amino-terminated PAMAM-G6 dendrimer is an effective antimicrobial agent against common Gram-negative and Grampositive pathogens. Hence, PAMAM-G6 could be an excellent candidate for a new class of antimicrobial compounds and could be incorporated to combat dominant bacteria in health care centers. 
fections is primarily due to the emergence of multidrugresistant strains (MDR), which is a worldwide health care concern (2). Approximate ly $90 \%$ of HCAI are caused by bacteria (3). The most important MDRs associated with these infections are methicillin resistant S. aureus (4), extended-spectrum beta-lactamase-producing E. coli (5), carbapenemase resistant $A$. buamanii (2), and P. aeruginosa (6). Therefore, considering the increasing prevalence of nosocomial infection by strains of MDR, the discovery and development of novel antibacterial agents, particularly those with structures and mechanisms of action different from traditional antibiotics, and a low potential to induce antibiotic resistance, now more than ever there is a need to control and treat health care- associated infections. Recently, the rapid growth in nanotechnology has spurred significant interest in the environmental applications of nanoparticles (NPs). NPs are excellent adsorbents, catalysts, and sensors due to their large specific surface area and high reactivity (7). More recently, several natural and engineered NPs including dendrimer NPs (8, 9), copper oxide (10), and zinc oxide (11) NPs have also been shown to have strong antimicrobial properties. However, dendrimers are a relatively new class of regularly branched macromolecules with unique structure and topological features that have emerged as strong antibacterial agents.

A typical dendrimer consists of a core molecule, monomeric branches called dendrons and surface functional groups that are able to react with other compounds (12). PAMAM dendrimers have been thoroughly explored for drug delivery and antimicrobial applications and have illustrated promising results, with amino-terminated dendrimers showing high antibacterial efficacy $(13,14)$. Their high antibacterial activity potential is attributed to the electrostatic interaction between the cationic dendrimer and the anionic bacteria cell surface with resultant disruption of the lipid bilayer, consequent cell lysis, and death. Thus, dendrimer biocides may be beneficial with respect to activity, localization in specific organs, reduced toxicity, and increased duration of action (13). An increase in the generation of PAMAM dendrimers is followed by a double increase in the number of functional amine groups in the structure of dendrimer (15). We designed a conceptional scheme of PAMAM-G6 dendrimer, which shows the number of functional amine groups in each generation (Fig. 1). Considering the fact that bacteria cause hospital infections and also considering the problem of MDR in bacteria, evaluating possible antibacterial properties in dendrimers and taking advantage of their ability as an antibacterial and antiseptic can be a research priority. The present study was conducted to determine the antibacterial properties of PAMAM-G6 dendrimer to remove the isolated bacteria from clinical specimens and standard strains bacteria. Overall, all the dominant bacteria related to HCAI have been investigated in the current study.

\section{Methods}

\section{Sampling and target bacteria}

In this study, sampling was performed in 16 hospitals and clinics in the city of Sabzevar, Iran, during August 2015 and May 2016. In total, 980 clinical samples including urine $(47 \%)$, blood $(27 \%)$, sputum $(13 \%)$, wounds $(8 \%)$, and burns $(5 \%)$ were collected from hospitals and clinics. Then, the samples were transferred into medical microbiology laboratory. Several smears were prepared from each sample and Gram and Giemsa staining were done simultaneously. Urine Samples were cultured in Mac Conkey Agar, blood agar, and Eosin methylene blue agar (EMB). Blood cultures of tryptic soy broth (TSB), Castaneda medium, and lysis centrifuge method were used. Also, Mac Conkey Agar and blood agar were used for sputum culture, and wounds samples were cultured in blood agar and chocolate agar. After 24 hours of incubation at $37^{\circ} \mathrm{C}$, differential catalase, oxidase, coagulase, indole, MRVP, TSI, SIM, citrate, and urease tests were per-

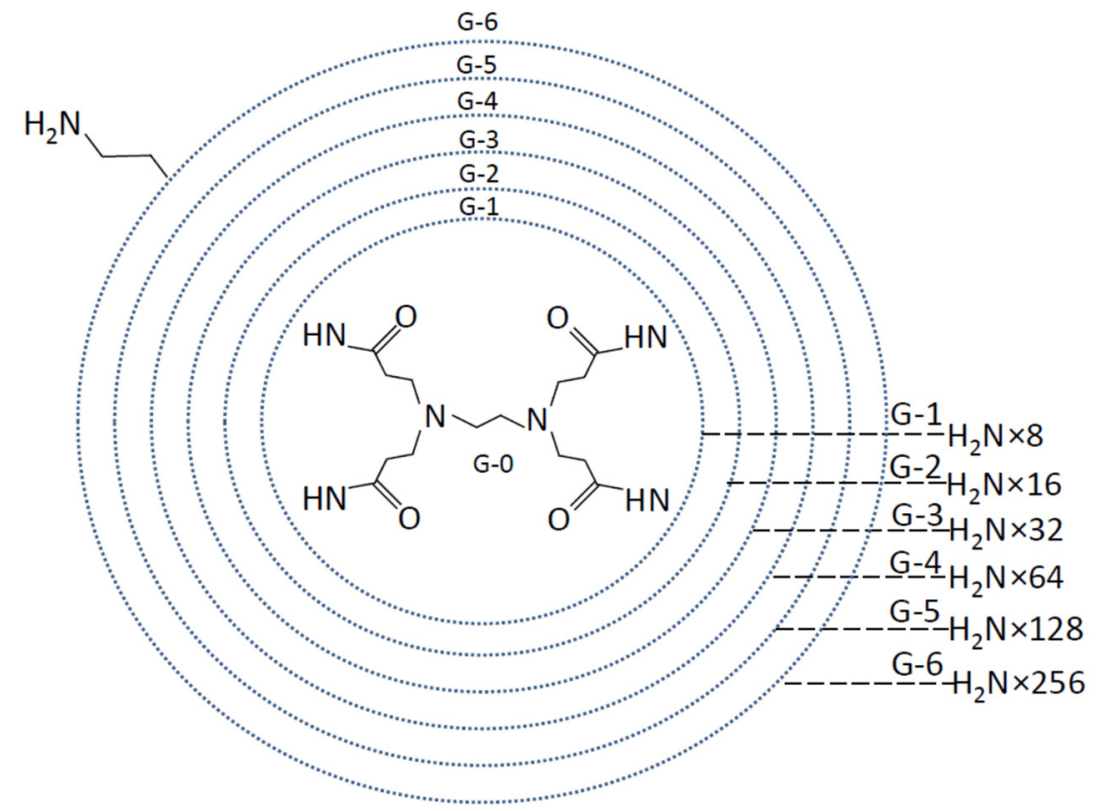

Fig. 1. Conception of PAMAM-G6 dendrimer 
formed to identify the bacterial species. The isolated bacteria included Gram-negative bacteria; $P$. aeruginosa, E. coli, A. baumannii, S. typhimurium, S. dysenteriae, $K$. pneumoniae, P. mirabilis, Gram-positive bacteria, and $S$. aureus and B. subtilis. Standard strains used in this study were E. coli ATCC 25922, P. aeruginosa ATCC 27853, A. baumannii ATCC 17957, S. typhimurium ATCC 19430, S. dysenteriae ATCC 13313, K. pneumoniae ATCC 1705, P. mirabilis ATCC 29906, S. aureus ATCC 25923, and B. subtilis ATCC 23857, which were provided by Iranian Research Organization for Science and Technology (IROST) and Pasteur Institute of Iran. Before use, all bacteria were cultured on specific media.

\section{Synthesis and properties of antimicrobial agent}

Tomalia's divergent growth approach is the most common method to synthesize ethylenediamine core PAMAM dendrimers (16). PAMAM-G6 was synthesized following a 2-step process, involving Michael addition of a suitable amine initiator core with methyl acrylate (MA), and exhaustive amidation of the resulting esters with large excess of ethylenediamine (EDA), reported elsewhere (17). Briefly, ethylenediamine $(10.0 \mathrm{~g}, 0.166 \mathrm{~mol})$ was dissolved in $100 \mathrm{~mL}$ methanol in a 1-1 round-bottomed flask. Methyl acrylate $(94.6 \mathrm{~g}, 0.751 \mathrm{~mol})$ was added at $40{ }^{\circ} \mathrm{C}$ and stirred for 24 hours in the presence of nitrogen exposure. Excess methyl acrylate was removed under vacuum condition at room temperature. A Michael addition between the amine and the acrylate yielded a product bearing 4 terminal methyl ester groups, defined as the G0.5 PAMAM. Subsequently, ethylenediamine $(120 \mathrm{~g}, 2.00 \mathrm{~mol})$ was dissolved in methanol and added to the G0.5 PAMAM. Then, a product bearing 4 terminal amino groups was obtained and defined as the G1 PAMAM after stirring for 48 hours in the presence of nitrogen. Moreover, by removing excess reactants using vacuum distillation, seventh generation PAMAM dendrimers was synthesized through repeating the above cycle. The chemical formula of PAMAMG6 is $\mathrm{C}_{2542} \mathrm{H}_{5088} \mathrm{~N}_{1018} \mathrm{O}_{508}$, molecular weight equal to $60713 \mathrm{~g} / \mathrm{mol}$, and the number of terminal amine groups is 256. Fourier transform infrared (FTIR, TENSOR 27 FTIR spectrometer, Bruker, Germany) was used to clarify the structural behavior of dendrimer on the molecular level. The samples were mixed with potassium bromide $(\mathrm{KBr})$ powder, and then the mixtures were made into pellet under high pressure. The sample pellet was scanned from 400 to $4000 \mathrm{~cm}^{-1}$. Pure $\mathrm{KBr}$ acted as blank. Morphology and size distribution of PAMAM-G6 was analyzed using transmission electron microscopy (TEM, Philips CM 30). For the TEM investigations, the samples were dispersed in ethanol and deposited by placing 2 drops of NPs suspension onto carbon-covered copper-grids, followed by drying at room temperature.

\section{Antimicrobial susceptibility testing}

Serial dilution of antimicrobial agent was prepared with using sterile distilled water to assess the antimicrobial activity of dendrimer. Clinical and Laboratory Standards Institute (CLSI) determined the antimicrobial activity of dendrimer by calculating minimum inhibitory concentra- tion (MIC) and minimum bactericidal concentrations (MBC) using the serial dilution method (18). Also, disc diffusion method was used to determinate the zone of inhibition.

\section{Disc diffusion method}

A bacteria culture that has been adjusted to $0.5 \mathrm{McFar}$ land standard was evenly applied to Mueller-Hinton agar (MHA) plates using a sterile swab. The volume of $25 \mu \mathrm{L}$ of antibacterial agent in different concentrations $(0.025$, $0.25,2.5$ and $25 \mu \mathrm{g} / \mathrm{mL}$ ) was impregnated to standard blank disc ( $6 \mathrm{~mm}$ in diameter). The discs were dried for 30 minutes and placed on the MHA seeded with the target culture and were incubated at $37^{\circ} \mathrm{C}$ for 24 hours.

\section{Experiments of MIC and $M B C$}

To determine the exact amount of MIC and MBC of bacteria, experiments with different concentrations $(0.025$, $0.25,2.5,25,50,100$ and $200 \mu \mathrm{g} / \mathrm{ml})$ of antimicrobial agent were conducted. Tubes containing $10 \mathrm{~mL}$ of nutrient broth (consisting of $10^{8} \mathrm{CFU} / \mathrm{mL}$ of bacteria and different concentrations of dendrimer) incubated in optimal conditions.

The positive control contained bacteria, with no dendrimers. Also, the negative control contained dendrimers in the absence of bacteria. A tube with the lowest concentration of antimicrobial agent and no bacterial growth was reported as MIC. To determine MBC, a loop of each tube with no growth transferred to nutrient agar plates and incubated in optimal conditions. A plate with no bacterial growth was taken as the MBC value.

\section{Cell cytotoxicity detection by MTT assay}

Cytotoxicity assessment was performed by MTT assay in HCT116cells (19) ( human intestinal cancer cell line), which was purchased from the Pasteur Institute Cell Bank of Iran (http://ncbi.pasteur.ac.ir/). Briefly, 5000 cells suspended in 96-well plates diluted in $100 \mu \mathrm{L}$ RPMI 1640 media (Invitrogen, Carlsbad, CA) were supplemented with $10 \%$ heat-inactivated fetal bovine serum (FBS, Invitrogen, Carlsbad, CA), and $100 \mathrm{mg} / \mathrm{mL}$ penicillinstreptomycin (Invitrogen, Carlsbad, CA) at $37{ }^{\circ} \mathrm{C}$ in a humidified atmosphere containing $5 \% \mathrm{CO}_{2}$. After 24 hours that all cells were attached to the baseline, $100 \mu \mathrm{L}$ of medium containing different concentration of PAMAM-G6 $(5,10,20,40,60,80$ and $100 \mu \mathrm{g} / \mathrm{mL})$ were added to each determined well and incubated in above conditions for 48 and 72 hours. One of the seeded wells in each repeat was used as a control in the absence of PAMAM-G6 dendrimer. After incubation, $20 \mu \mathrm{L}$ containing $5 \mathrm{mg} / \mathrm{mL}$ MTT were added to the wells and incubated again for 4 hours. During this time, the MTT (yellow tetrazolium salt) was enzymatically converted into the purple formazan precipitate by viable cells, and the concentration of formazan showed the proportion of viable cells. Subsequently, all media were aspirated from the cells, then, $150 \mu \mathrm{L}$ of DMSO was added to dissolve the formazan. Finally, absorbance was detected at $490 \mathrm{~nm}$ wavelength by ELISA plate reader. 


\section{Data analysis}

Statistical analysis was performed using Mann-Whitney $\mathrm{U}$ test analysis. Statistical significance was set at $\mathrm{p}<0.05$. Every experiment was repeated at least 3 times.

\section{Results}

As demonstrated in Fig. 2, the isolated bacteria were $E$. coli $(23.65 \%)$, S. aureus $(24.7 \%)$, P. aeruginosa $(10.9 \%)$, B. subtilis (7.7\%), S. typhimurium (8.87\%), A. baumannii (7.02\%), K. pneumoniae (7.1\%), P. mirabilis $(6.46 \%)$, and $S$. dysenteriae (3.6\%). Fig. 2 displays that the frequently of the isolated bacteria with the highest percentage (46.4\%) were E. coli, $S$. aureus, and P. aeruginosa from urine and blood samples. In addition, these 3 bacteria were present in most urine, blood, sputum, wounds, and burns samples. In other words, $59.25 \%$ of all isolated species belong to these 3 bacteria. FTIR analysis of PAMAM-G6 NPs was performed to confirm the existence of characteristic amides, terminal amino, and etc. (Fig. 3). Results in
Table 1 demonstrate the corresponding functional groups of the wavelengths, which are indicated in Fig. 3. The morphology and diameter of PAMAM-G6 NPs were studied by TEM as shown in Fig. 4. PAMAM-G6 NPs were shown to have a spherical shape with a mean diameter size of $20 \mathrm{~nm}$. The results of different concentration of PAMAM-G6 dendrimer effect on isolated bacteria and standard strain (using disk diffusion method) are presented in Tables 2 and 3, respectively. According to the obtained results, PAMAM-G6 dendrimer actively inhibited the growth of isolated Gram- negative and Gram- positive bacteria and standard strains. However, the antibacterial activity of PAMAM-G6 on the isolated bacteria was less than that on the standard strains, whose difference was statistically significant $(p<0.05)$. The most sensitivity was related to $P$. mirabilis ATCC 29906, S. typhimurium ATCC 19430, S. dysenteriae ATCC 13313, and $S$. aureus ATCC 25923 at the concentration of $25(\mu \mathrm{g} / \mathrm{mL})$ PAMAM-G6 with the inhibition zone of $35,32,31$, and 30 $\mathrm{mm}$, respectively. In addition, the least sensitivity was

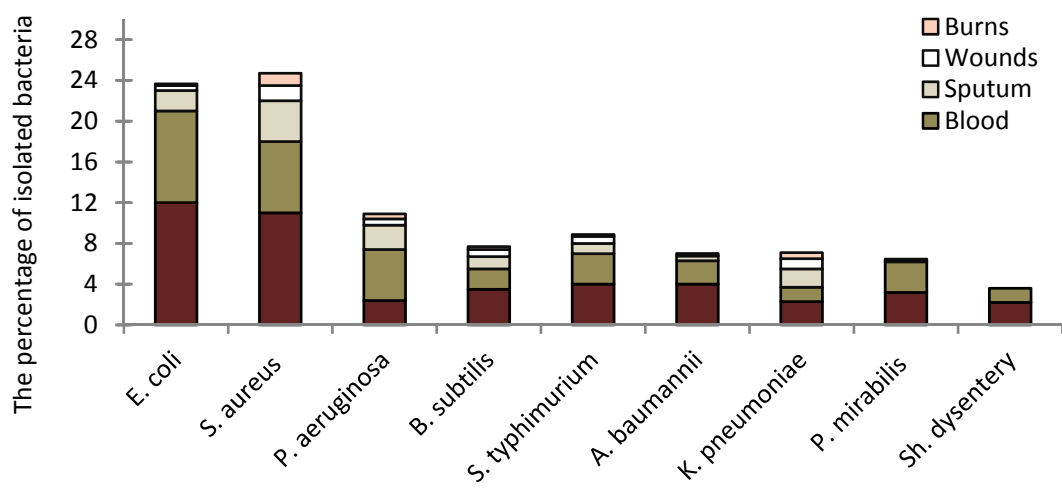

Type of isolated bacteria

Fig. 2. Type of Isolated Bacteria and the Frequency Distribution of Each Bacterium in Urine, Blood, Sputum, Wounds and Burns Samples

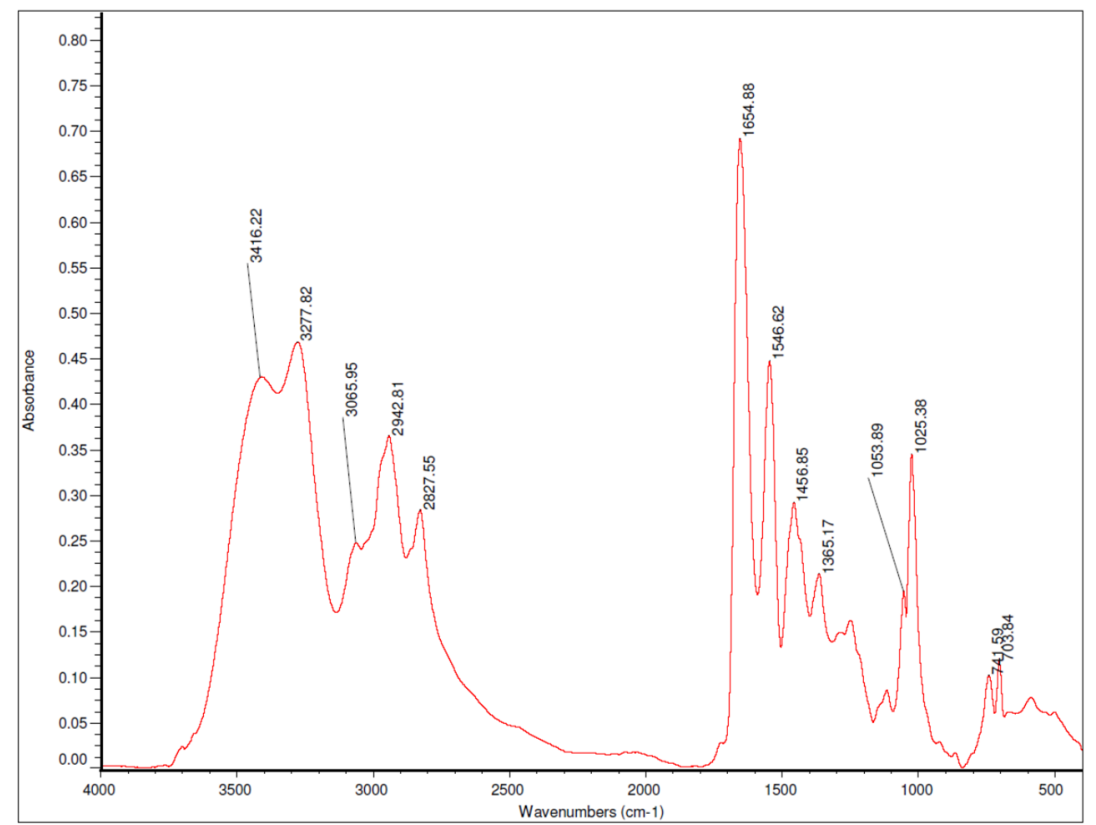

Fig. 3. FTIR Spectra of PAMAM-G6 
Table 1. Band position of PAMAM-G6 spectrum

\begin{tabular}{ll}
\hline Wavenumber $\left(\mathrm{cm}^{-1}\right)$ & Spectral assignments \\
\hline 1025.38 & $\mathrm{C}-\mathrm{O}$ stretching vibration \\
1654.88 & $\mathrm{C}=\mathrm{O}$ stretching (amide I) \\
1546.62 & $\mathrm{~N}-\mathrm{H}$ bending/C-N stretching (amide II) \\
1456.85 & H-C-H scissor \\
1365.17 & H-C-H asymmetric \\
2827.55 and 2942.81 & $\mathrm{C}-\mathrm{H}$ stretching vibrations \\
$3416.22 \mathrm{~cm}^{-1}$ and 3277.82 & $\mathrm{~N}-\mathrm{H}$ stretching mode of amineI and amide groups \\
\hline
\end{tabular}

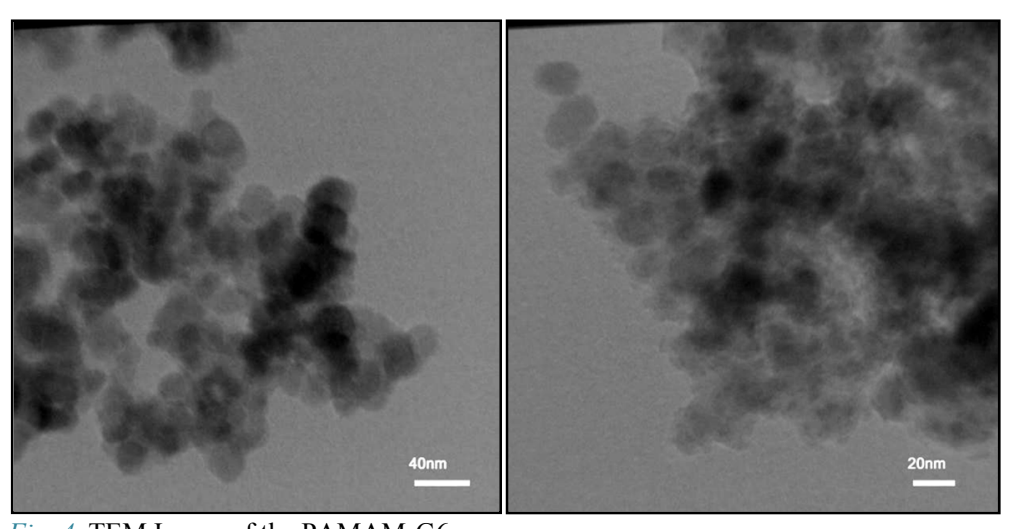

Fig .4. TEM Image of the PAMAM-G6

related to A. baumannii at $25(\mu \mathrm{g} / \mathrm{mL})$ concentration of dendrimer, with $10 \mathrm{~mm}$ zone of inhibition. The concentration of $0.025(\mu \mathrm{g} / \mathrm{ml})$ dendrimer had no effect on the studied bacteria, except $S$. typhimurium, $P$. mirabilis, and $S$. aureus. Comparison between MIC and MBC values of PAMAM-G6 for isolated bacteria and standard strains are presented in Tables 4 and 5, respectively. As can be seen in the tables, the maximum amount of MIC and MBC were related to isolated bacteria, ie, E. coli and A. baumannii at 25 and $200 \mu \mathrm{g} / \mathrm{mL}$ concentrations of dendrimer, respectively. Also, the minimum amount of MIC and MBC were related to S. typhimurium ATCC 19430 at 0.025 and $25 \mu \mathrm{g} / \mathrm{mL}$ concentrations, respectively. The effect of PAMAM-G6 on cell viability was assessed using MTT assay. This assay measured optical density of different concentrations in comparison with the rate of viable cells. As displayed in Fig. 5, there were little cytotoxicity effects of PAMAM-G6 with different concentrations in HCT 116 cells during 48 hours when no IC50 value was obtained (Graph pad prism 6, USA). The cytotoxicity effects after 72 hours (Fig. 5) were more than 48 hours, with no measurable IC50. However, high concentration in 72 hours had more effects compared to the same concentration in 48 hours.

\begin{tabular}{|c|c|c|c|c|c|c|c|c|c|}
\hline \multirow[t]{2}{*}{ Dendrimer concentration, $\mu \mathrm{g} / \mathrm{ml}$} & \multicolumn{9}{|c|}{ Zone of inhibition, $\mathrm{mm}$} \\
\hline & $\begin{array}{l}\dot{\delta} \\
0 \\
0\end{array}$ & 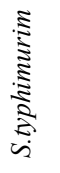 & 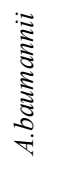 & 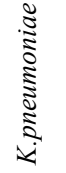 & 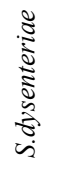 & 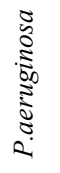 & 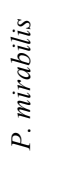 & 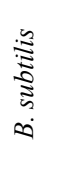 & 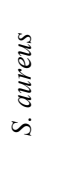 \\
\hline 0.025 & 0 & 10 & 0 & 0 & 0 & 0 & 9 & 0 & 9 \\
\hline 0.25 & 0 & 19 & 0 & 13 & 0 & 0 & 19 & 9 & 10 \\
\hline 2.5 & 0 & 21 & 0 & 16 & 13 & 0 & 21 & 9 & 11 \\
\hline 25 & 20 & 31 & 10 & 26 & 27 & 22 & 32 & 17 & 21 \\
\hline
\end{tabular}

Table 3. The Mean Diameter of Inhibition Zone Of Standard Strain Bacteria VS Different Concentrations of PAMAM-G6 Dendrimer Dendrimer concentration, $\mu \mathrm{g} / \mathrm{ml}$ Zone of inhibition, $\mathrm{mm}$

\begin{tabular}{|c|c|c|c|c|c|c|c|c|c|}
\hline & 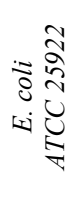 & 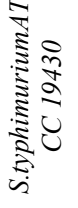 & 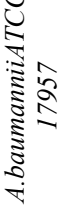 & 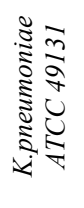 & 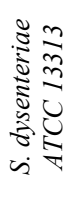 & 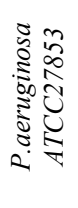 & 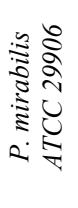 & 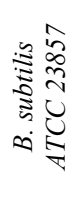 & 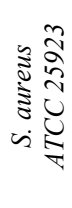 \\
\hline 0.025 & 0 & 14 & 0 & 0 & 0 & 0 & 11 & 0 & 11 \\
\hline 0.25 & 0 & 22 & 9 & 19 & 14 & 0 & 21 & 9 & 11 \\
\hline 2.5 & 9 & 23 & 13 & 21 & 18 & 9 & 22 & 9 & 17 \\
\hline 25 & 24 & 32 & 20 & 27 & 30 & 22 & 35 & 21 & 30 \\
\hline
\end{tabular}




\begin{tabular}{|c|c|c|c|c|c|c|c|c|c|}
\hline & $\begin{array}{l}\dot{8} \\
\dot{0} \\
\dot{i}\end{array}$ & 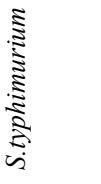 & 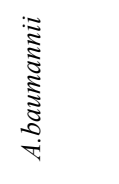 & 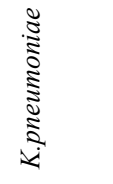 & 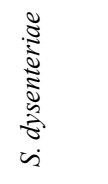 & 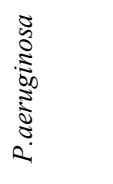 & 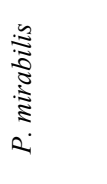 & 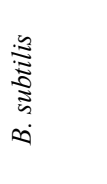 & $\begin{array}{l}\tilde{a} \\
\vdots \\
\vdots \\
\vdots \\
\dot{n}\end{array}$ \\
\hline 0.025 & Growth & Growth & Growth & Growth & Growth & Growth & Growth & Growth & Growth \\
\hline 0.25 & Growth & MIC & Growth & MIC & MIC & Growth & MIC & MIC & MIC \\
\hline 2.5 & Growth & B.S & Growth & B.S & B.S & MIC & B.S & B.S & B.S \\
\hline 25 & MIC & MBC & MIC & B.S & B.S & B.S & B.S & B.S & B.S \\
\hline 50 & B.S & B.C** & B.S & B.S & MBC & B.S & B.S & MBC & B.S \\
\hline 100 & B.S & B.C & B.S & $\mathrm{MBC}$ & B.C & $\mathrm{MBC}$ & $\mathrm{MBC}$ & B.C & $\mathrm{MBC}$ \\
\hline 200 & $\mathrm{MBC}$ & B.C & $\mathrm{MBC}$ & B.C & B.C & B.C & B.C & B.C & B.C \\
\hline \multicolumn{10}{|l|}{ B.S*: Bacteriostatic, B.C C $^{* *}$ : Bactericide } \\
\hline \multirow[b]{2}{*}{ Dendrimer concentration, $\mu \mathrm{g} / \mathrm{ml}$} & \multicolumn{9}{|c|}{ Type of Effect } \\
\hline & 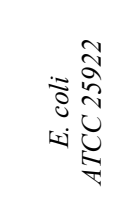 & 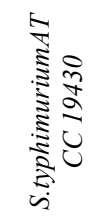 & 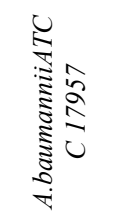 & 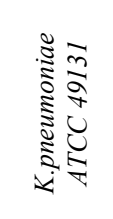 & 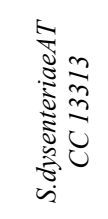 & 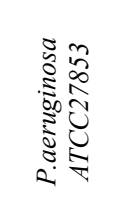 & 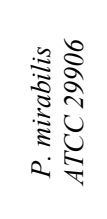 & 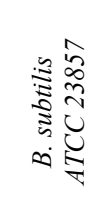 & 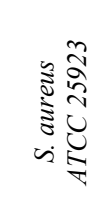 \\
\hline 0.025 & Growth & MIC & Growth & Growth & Growth & Growth & Growth & Growth & Growth \\
\hline 0.25 & Growth & B.S & Growth & MIC & MIC & Growth & MIC & MIC & MIC \\
\hline 2.5 & MIC & $\mathrm{MBC}$ & MIC & B.S & B.S & MIC & B.S & B.S & B.S \\
\hline 25 & B.S & B.C & B.S & B.S & B.S & B.S & B.S & B.S & B.S \\
\hline 50 & B.S & B.C & B.S & B.S & MBC & B.S & B.S & MBC & B.S \\
\hline 100 & $\mathrm{MBC}$ & B.C & B.S & MBC & B.C & $\mathrm{MBC}$ & $\mathrm{MBC}$ & B.C & $\mathrm{MBC}$ \\
\hline 200 & B.C & B.C & $\mathrm{MBC}$ & B.C & B.C & B.C & B.C & B.C & B.C \\
\hline
\end{tabular}

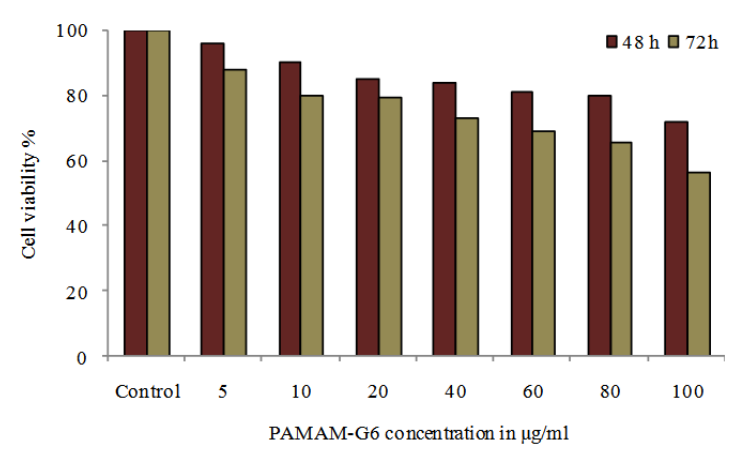

Fig. 5. Cytotoxicity of PAMAM-G6 in HCT116 Cells. Cell viability was determined by MTT assay in HCT116 cells in $48 \mathrm{~h}$ and $72 \mathrm{~h}$. As depicted in this figure there are cytotoxicity effects in different concentration of PAMAM-G6 dendrimer during $48 \mathrm{~h}$ and $72 \mathrm{~h}$ which it is high level in $72 \mathrm{~h}$. The IC50 was not determined in both times. The reported values were carried out two independent experiments with 3 repeated times and untreated cells were assumed to be viable as control cells.

\section{Discussion}

A well-known problem in modern-day hospitalization is the occurrence of nosocomial or health care acquired infections caused by multi-resistant pathogens (3). PAMAM dendrimers have been investigated for their biological applications, but antibacterial activity has not been extensively explored (20). The 980 samples were tested. Fig. 2 shows the isolated bacteria with the highest frequency belonging to E. coli (23.65\%), S. aureus (24.7\%), and P. aeruginosa $(10.9 \%)$. These 3 bacteria were mostly isolated from urine and blood samples as $46.4 \%$ of these bacteria were isolated from these samples; and of these 9 isolated strains, the highest frequency belonged to these 3 bacteria $(59.25 \%)$. Similar results were observed by Sala- mati et al. (21), as they reported that the most common pathogenic organisms were Enterobacter (27\%), S. aureus (21\%), and E.coli (14\%). Other studies also reported that the most common nosocomial infections were associated with E.coli (22), P. aeruginosa, and S. aureus (23). In a study conducted by Mazloomi et al. (24), on 172 specimens collected at a children medical center, the most frequent isolated bacteria were $S$. aureus and P. aeruginosa.

Ferreira et al. (25) investigated patients' urine and blood specimens and observed that the highest frequency of bacteria in urine and blood specimen belonged to $E$. coli and $S$. aureus, respectively. These pathogens, especially $E$. coli, $P$. aeruginosa, $S$. aureus, A. baumannii, and $K$. pneumoniae, are often HCAIs such as Bacterimia, UTIs, 
pneumonia, and etc. (26). The size of the inhibition zone clearly shows that with increasing concentration of the antimicrobial agent, the zone surrounding the disks is expanded, which indicates the power of antimicrobial agent at higher concentrations. According to our findings, PAMAM derivatives could be considered as an excellent candidate for a new class of antimicrobial compounds that could be incorporated to combat health care-acquired infections. According to minimum bacteriostatic or bactericide levels (Tables 4 and 5), it is clear that PAMAM-G6 has antimicrobial effects and can be used as an antimicrobial agent. Previous studies have shown that antimicrobial agents cause bacterial cell membrane damage, spatial deformation, degradation of bacterial enzymes, and damage of chromosome and bacteria cell wall damage. This character refers to end amine groups in dendrimer structure, which interact with the negative charge of the membrane or cytoplasm microorganism, causing bacterial cell wall damage, and finally, inactivation of bacteria (27). In FTIR spectrum of PAMAM-G6 dendrimer (Fig. 3), 9 main peaks are detectable at $1025 \mathrm{~cm}^{-1}, 1365 \mathrm{~cm}^{-1}, 1456 \mathrm{~cm}^{-1}$, $1546 \mathrm{~cm}^{-1}, 1654 \mathrm{~cm}^{-1}, 2827 \mathrm{~cm}^{-1}, 2942 \mathrm{~cm}^{-1} 3277 \mathrm{~cm}^{-1}$, and $3416 \mathrm{~cm}^{-1}$, with the last peak being related to N-H stretching vibration of primary amine. Other main band positions, based on wave number and their assignments are presented in Table 1. Overall, PAMAM-G6 dendrimer is an efficient antibacterial agent against both Gram-negative and Gram-positive bacteria. Our results are also consistent with those of Lopez et al. (28) study. They found that the antimicrobial effect of PAMAM dendrimers was modified with amine groups on Gram-positive and Gram-negative bacteria. Likewise, Xue et al. and Charles et al. found that amino-terminated PAMAM G2 and G3 dendrimers possess significant antibacterial effects against multi-resistant strains $(12,29)$. As shown in Tables 2 to 5, E. coli, P. aeruginosa, and A. baumannii had a higher resistance than other studied bacteria. Intrinsic and acquired resistance of these bacteria against the antibacterial agent can be attributed to the possible effect of lower concentrations of dendrimer PAMAM-G6 on the bacteria such as E. coli, $P$. aeruginosa (30), and A. baumannii (31) than other target bacteria. Mihani. et al. indicated that $P$. aeruginosa were resistant against ciprofloxacin (67\%), ceftazidime (71\%), and imipenem (41\%) (32). Mohammadimehr et al. (33), also, showed that $E$. coli has a high resistance against ampicillin, amikacin and piperacillin inoculated discs. The findings of the present study are consistent with their results. The antibacterial activity of PAMAM-G6 on the isolated bacteria was less than that on the standard strains (Tables 2-5). For example, according to Table 4 and 5, the MIC of PAMAM-G6 related to E. coli and A. baumannii was $25 \mu \mathrm{g} / \mathrm{mL}$ and the MIC of PAMAM-G6 was related to E. coli ATCC 25922, and A. baumannii ATCC 17957 was $2.5 \mu \mathrm{g} / \mathrm{mL}$. High resistance of bacteria isolated from hospital than standard strains can be attributed to the acquired resistance. Many studies also confirm that most bacteria in hospitals have been resistant towards most anti-bacterial materials. According to reports, most of A. baumannii isolated from clinical specimens were resistant to ciprofloxacin positions in the top $85 \%$ (34). A study conducted to evaluate the resistance of $P$. aeruginosa towards ceftazidime showed that in Lithuania $78.9 \%$ (35) has developed resistance to ceftazidime. The diameter of inhibition zone for $S$. typhimurium, $P$. mirabilis, and $S$. aureus was also observed at the concentration of $0.025 \mu \mathrm{g} / \mathrm{mL}$ of dendrimer PAMAM-G6 (Tables 3-4). The study conducted by Izanlo et al. (36) on the effect of PAMAM-G4 dendrimer on E. coli, Enterobacter cloacae, B. subtilis and $S$. aureus by means of disc diffusion method concluded that concentration of $0.05 \mu \mathrm{g} / \mathrm{mL}$ has no effect on these bacteria and also they found that the antibacterial effect of PAMAM-G4 occurs at higher concentrations. Izanlo et al. (37) examined the effect of dendrimer PAMAM-G4 on Klebsiella oxytoca, $P$. mirabilis, and $P$. aeruginosa using disc diffusion method and found that concentrations of $0.5,5$, and $50 \mu \mathrm{g} / \mathrm{mL}$ of PAMAM-G4 have no effect on these selected bacteria. Perhaps, the higher antibacterial effect of dendrimerPAMAM-G6, compared with lower generation dendrimers, can be attributed to high density, ordered and hyperbranching structure, high spatial void between branches, large number of terminal functional groups, and relatively large molecular size of PAMAMG6 (20). These characteristics lead to highly specific area in dendrimer PAMAM-G6, which causes higher activity of dendrimers in the surface of culture and higher efficiency at lower concentrations. However, most importantly, it is the number of terminal amine groups of dendrimer for generation 4 is 64, while the number of terminal amine groups for PAMAM-G6 is 256 (38). These functional groups are adsorbed on the bacterial cell surfaces, diffused through the cell wall, and bonded to cytoplasmic membrane; and as a result, release electrolytes such as potassium ions and phosphate from the cell and also nucleic materials such as DNA and RNA due to disruption and disintegrate of the cytoplasmic membrane. Therefore, it is proposed that the antibacterial property of dendrimers be mediated by disrupting the bacterial outer and inner membrane by terminal amine groups (27). We also measured the cytotoxicity of PAMAM-G6 on the HCT 116 cell line, and the data revealed that the cytotoxicity will be increased in cells at higher concentrations and in longterm treatments. Mukherjee et al. studied cytotoxicity in different generations of PAMAM dendrimers with variety of doses. They found that increasing the dose of these dendrimers cause a decrease in the percentage of healthy and early apoptotic cells. They also demonstrated that the systematic mechanism in mammalian cells leads to cytotoxicity in various exposures of different generations of dendrimers. In line with a recent study, our data revealed a reduction in cell viability although the dose and exposure time are 2 important factors. This result is consistent with a study that found PAMAM could lead to the formation of nanoscale holes in eukaryotic membranes at high concentrations (39). Increasing the number of surface amino groups in the dendrimers and zeta potential may give rise to toxicity after increasing generation and diameter (19).

\section{Conclusion}

This study evaluated the antibacterial effects of PAMAM-G6 on the isolated bacteria from clinical specimens 
and standard strains using MIC, MBC, and disc diffusion method. The highest number of isolated bacteria was related to E. coli $(23.65 \%)$, S. aureus $(28.7 \%)$, and P. aeruginosa $(10.9 \%)$. In this study, we found that aminoterminated PAMAM-G6 dendrimer is an effective antimicrobial agent against common Gram-negative and Grampositive pathogens. Bacteria origination is one of the important variables affecting the performance of PAMAMG6. The antibacterial activity of PAMAM-G6 on the isolated bacteria was less than that on the standard strains. Although increasing the concentration of PAMAM-G6 improves removal efficiency, their cytotoxicity in mammalian cells has to be considered at higher concentrations. However, the low levels have relatively high antibacterial effects on Gram- negative and Gram- positive bacteria. $A$. baumannii has the least sensitivity to PAMAM-G6, where P. mirabilis ATCC 29906, S. typhimurium ATCC 19430, S. dysenteriae ATCC 13313, and S. aureus ATCC 25923 have the highest sensitivity. These findings indicated that PAMAM-G6 could be an excellent candidate for a new class of antimicrobial compounds and could be incorporated to combat dominant bacteria in health care centers.

\section{Acknowledgements}

The authors gratefully acknowledge all the support for this study that was provided by the School of Public Health, Sabzevar University of Medical Sciences, Sabzevar, Iran.

\section{Funding}

This work was funded by School of Public Health, Sabzevar University of Medical Sciences, Sabzevar, Iran (Grant number: 394040415).

\section{Conflict of Interests}

The authors declare that they have no competing interests.

\section{References}

1. Broex E, Van Asselt A, Bruggeman C, Van Tiel F. Surgical site infections: how high are the costs? Journal of Hospital Infection. 2009; 72(3): 193-201.

2. Rajamohan G, Srinivasan V, Gebreyes W. Biocide-tolerant multidrugresistant Acinetobacter baumannii clinical strains are associated with higher biofilm formation. Journal of Hospital Infection. 2009;73(3):287-9.

3. LI Ly, JIA HX, JIA JX, ZHAO XL, ZHAO YC, REN JH, et al. Study on effect of infection control of multidrug-resistant bacteria infections in general hospital [J]. Chinese Journal of Nosocomiology. 2011;20:056.

4. Porter E, Damani N. Epidemic meticillin-resistant Staphylococcus aureus strains associated with Northern Ireland. Journal of Hospital Infection. 2007;65(1):88-9.

5. Banerjee R, Robicsek A, Kuskowski MA, Porter S, Johnston BD, Sokurenko E, et al. Molecular epidemiology of Escherichia coli sequence type 131 and its $\mathrm{H} 30$ and H30-Rx subclones among extended-spectrum- $\beta$-lactamase-positive and-negative E. coli clinical isolates from the Chicago region, 2007 to 2010. Antimicrobial agents and chemotherapy. 2013;57(12):6385-8.

6. Zavascki AP, Carvalhaes CG, Picão RC, Gales AC. Multidrugresistant Pseudomonas aeruginosa and Acinetobacter baumannii: resistance mechanisms and implications for therapy. Expert review of anti-infective therapy. 2010; 8(1): 71-93.

7. Li Q, Mahendra S, Lyon DY, Brunet L, Liga MV, Li D, et al. Antimicrobial nanomaterials for water disinfection and microbial control: potential applications and implications. Water research. 2008; 42 (18): 4591-602.

8. Gholami M, Nazari S, Farzadkia M, Mohseni SM, Alizadeh Matboo S, Akbari Dourbash F, et al. Nano polyamidoamine-G7 dendrimer synthesis and assessment the antibacterial effect in vitro. Tehran University Medical Journal. 2016; 74(1): 25-35.

9. Gholami M, Nazari S, Farzadkia M, Majidi G, Alizadeh Matboo S. Assessment of nanopolyamidoamine-G7 dendrimer antibacterial effect in aqueous solution. Tehran University Medical Journal. 2016; 74(3): 159-67.

10. Ren G, Hu D, Cheng EW, Vargas-Reus MA, Reip P, Allaker RP. Characterisation of copper oxide nanoparticles for antimicrobial applications. International journal of antimicrobial agents. 2009; 33(6): 587-90.

11. Sirelkhatim A, Mahmud S, Seeni A, Kaus NHM, Ann LC, Bakhori SKM, et al. Review on zinc oxide nanoparticles: antibacterial activity and toxicity mechanism. Nano-Micro Letters. 2015;7(3): 219-42.

12. Xue X, Chen X, Mao X, Hou Z, Zhou Y, Bai H, et al. Aminoterminated generation 2 poly (amidoamine) dendrimer as a potential broad-spectrum, nonresistance-inducing antibacterial agent. The AAPS journal. 2013; 15(1): 132-42.

13. Calabretta MK, Kumar A, McDermott AM, Cai C. Antibacterial activities of poly (amidoamine) dendrimers terminated with amino and poly (ethylene glycol) groups. Biomacromolecules. 2007; 8(6): 180711.

14. Menjoge AR, Kannan RM, Tomalia DA. Dendrimer-based drug and imaging conjugates: design considerations for nanomedical applications. Drug discovery today. 2010; 15(5): 171-85.

15. Zhu W, Okollie B, Bhujwalla ZM, Artemov D. PAMAM dendrimer-based contrast agents for MR imaging of Her-2/neu receptors by a three-step pretargeting approach. Magnetic resonance in medicine. 2008; 59(4): 679-85.

16. Esfand R, Tomalia, DA. Laboratory synthesis of poly (amidoamine) (PAMAM) dendrimers. In: Frechet JMJ, Tomalia DA. (Eds.), Dendrimers and Other Dendritic Polymers. John Wiley, New York. 2001, pp. 589-604.

17. Tomalia DA, Baker H, Dewald J, Hall M, Kallos G, Martin S, et al. Dendritic macromolecules: synthesis of starburst dendrimers Macromolecules. 1986; 19: 2466-8.

18. Clinical Laboratory Standards Institute (CLSI). CLSI Document M100S-S22. Performance Standards for Antimicrobial Susceptibility Testing: Twenty Third Informational Supplement ed. Wayne: CLSI; 2012.

19. Mukherjee SP, Lyng FM, Garcia A, Davoren M, Byrne HJ. Mechanistic studies of in vitro cytotoxicity of poly (amidoamine) dendrimers in mammalian cells. Toxicology and applied pharmacology. 2010; 248(3): 259-68.

20. Felczak A, Wrońska N, Janaszewska A, Klajnert B, Bryszewska M, Appelhans D, et al. Antimicrobial activity of poly (propylene imine) dendrimers. New Journal of Chemistry. 2012; 36 (11): 2215-22.

21. Salamati P, Rahbarimanesh AA, Yunesian M, Naseri M. Neonatal nosocomial infections in Bahrami children hospital. The Indian Journal of Pediatrics. 2006; 73(3): 197-200.

22. Walther B, Luebke-Becker A, Stamm I, Gehlen H, Barton AK, Janssen $\mathrm{T}$, et al. Suspected nosocomial infections with multi-drug resistant E. coli, including extended-spectrum beta-lactamase (ESBL)producing strains, in an equine clinic. Berliner und Munchener tierarztliche Wochenschrift. 2013;127(11-12): 421-7.

23. Tarr PI, Warner BB, editors. Gut bacteria and late-onset neonatal bloodstream infections in preterm infants. Seminars in Fetal and Neonatal Medicine; 2016: Elsevier.

24. Nobandegani NM, Mahmoudi S, Pourakbari B, Sadeghi RH, Sani MN, Farahmand F, et al. Antimicrobial susceptibility of microorganisms isolated from sputum culture of patients with cystic fibrosis: Methicillin-resistant Staphylococcus aureus as a serious concern. Microbial Pathogenesis. 2016; 100: 201-4.

25. Ferreira L, Sánchez-Juanes F, Muñoz-Bellido J, González-Buitrago J. Rapid method for direct identification of bacteria in urine and blood culture samples by matrix-assisted laser desorption ionization time-of-flight mass spectrometry: intact cell vs. extraction method. Clinical Microbiology and Infection. 2011; 17(7): 1007-12.

26. Mithraja MJ, Irudayaraj V, Kiruba S, Jeeva S. Antibacterial efficacy of Drynaria quercifolia (L.) J. Smith (Polypodiaceae) against clinically isolated urinary tract pathogens. Asian Pacific Journal of Tropical Biomedicine. 2012; 2(1): S131-S5. 
27. Wang B, Navath RS, Menjoge AR, Balakrishnan B, Bellair R, Dai H, et al. Inhibition of bacterial growth and intramniotic infection in a guinea pig model of chorioamnionitis using PAMAM dendrimers. International journal of pharmaceutics. 2010;395(1):298-308.

28. Lopez AI, Reins RY, McDermott AM, Trautner BW, Cai C. Antibacterial activity and cytotoxicity of PEGylated poly (amidoamine) dendrimers. Molecular BioSystems. 2009; 5(10): 114856.

29. Charles S, Vasanthan N, Kwon D, Sekosan G, Ghosh S. Surface modification of poly (amidoamine)(PAMAM) dendrimer as antimicrobial agents. Tetrahedron letters. 2012; 53(49): 6670-5.

30. Breidenstein EB, de la Fuente-Núñez C, Hancock RE. Pseudomonas aeruginosa: all roads lead to resistance. Trends in microbiology. 2011;19(8): 419-26.

31. Rajamohan G, Srinivasan VB, Gebreyes WA. Molecular and functional characterization of a novel efflux pump, AmvA, mediating antimicrobial and disinfectant resistance in Acinetobacter baumannii. Journal of antimicrobial chemotherapy. 2010; 65(9): 1919-25.

32. Mihani F KA. MBL-producing Pseudomonas aeruginosa strains isolated from patients with burn wound infections and PCR methods to identify blaVIM , blaIMP genes. Iran J Microbiol. 2007; 1(1): 23-31.

33. Mohammadimehr M, Feizabadi M, Bahadori A. Antibiotic resistance pattern of Gram negative bacilli caused nosocomial infections in ICUs in khanevadeh and golestan hospital in Tehran-2007. Annals of military and health sciences research. 2011; 8: 283-290.

34. Lagamayo EN. Antimicrobial resistance in major pathogens of hospital-acquired pneumonia in Asian countries. American journal of infection control. 2008; 36(4): S101-S8.

35. Gailiene G, Pavilonis A, Kareivienè V. The peculiarities of Pseudomonas aeruginosa resistance to antibiotics and prevalence of serogroups. Medicina (Kaunas). 2007; 43(1): 36-42.

36. Izanloo H, Ahmadi Jebelli M, Nazari Sh, Safavi N, Tashauoei HR, Majidi Gh, et al. Studying the antibacterial effect of polyamidoamineG4 dendrimer on some of the Gram-negative and Gram-positive bacteria. J Arak Univ Med Sci. 2014;17:1-10 (Full Text in Persion)

37. Izanloo H, Ahmadi Jabali M, Tashyiee H, Khazaee M, Nazari Sh, Majidi Gh, et al. The antimicrobial effects of Polypropylenimine-G2 and Polyamidoamine-G4 dendrimers on Klebsiella oxytoca, Pseudomonas aeruginosa and Proteus mirabilis, in vitro experiment. J Sabzevar Univ Med Sci. 2014; 21: 925-33 (Full Text in Persion).

38. Hermanson GT. Bioconjugate techniques: Third ed: Elsevier; 2013. p. 351-86.

39. Hong S, Leroueil PR, Janus EK, Peters JL, Kober M-M, Islam MT, et al. Interaction of polycationic polymers with supported lipid bilayers and cells: nanoscale hole formation and enhanced membrane permeability. Bioconjugate chemistry. 2006; 17(3): 728-34. 